01

\title{
Сужение линии поглощения легких атомов щелочных металлов в атмосфере тяжелых инертных газов при росте интенсивности излучения
}

\author{
(C) А.И. Пархоменко ${ }^{1}$, А.М. Шалагин ${ }^{1,2}$ \\ ${ }^{1}$ Институт автоматики и электрометрии Сибирского отделения РАН, \\ 630090 Новосибирск, Россия \\ ${ }^{2}$ Новосибирский государственный университет, \\ 630090 Новосибирск, Россия \\ e-mail: par@iae.nsk.su, shalagin@iae.nsk.su \\ Поступила в редакцию 19.03.2019 г. \\ В окончательной редакции 31.05.2019 г. \\ Принята к публикации 11.06.2019 г.
}

Исследован эффект сужения линии поглощения легких атомов щелочных металлов ${ }^{7} \mathrm{Li}$ и ${ }^{23} \mathrm{Na}$, находящихся в атмосфере тяжелого инертного газа Хе, при росте интенсивности внешнего излучения. Для атомов ${ }^{7} \mathrm{Li}$ при температуре $T=300 \mathrm{~K}$ и давлении буферного газа ксенона $p_{\text {Хе }}=0.002$ Torr ширина линии на полувысоте уменьшается в 1.20 раза при росте интенсивности излучения от $1 \mu \mathrm{W} / \mathrm{cm}^{2}$ до $2.5 \mathrm{~mW} / \mathrm{cm}^{2}$. Для атомов ${ }^{23} \mathrm{Na}$ при $T=600 \mathrm{~K}$ и $p_{\text {Хе }}=0.01$ Torr ширина линии на полувысоте уменьшается в 1.29 раза при росте интенсивности излучения от $1 \mu \mathrm{W} / \mathrm{cm}^{2}$ до $6 \mathrm{~mW} / \mathrm{cm}^{2}$. Эффект полевого сужения линии поглощения обусловлен, во-первых, тем, что столкновительная релаксация скоростей легких резонансных частиц в атмосфере тяжелых буферных частиц разбивается на два существенно различных по продолжительности этапа: релаксация по направлению скорости (быстрый этап) и релаксация по модулю скорости (медленный этап), и во-вторых, отсутствием столкновительных переходов между сверхтонкими компонентами основного состояния.

Ключевые слова: литий, натрий, буферный газ, столкновения, линия поглощения.

DOI: $10.21883 /$ OS.2019.11.48505.118-19

\section{Введение}

В спектроскопии атомов одной из важнейших характеристик исследуемого объекта является ширина линии поглощения излучения. Хорошо известно, что с ростом интенсивности излучения ширина линии увеличивается вследствие эффекта насыщения (полевое уширение) [13]. В теоретической работе [4] обращено внимание на возможность принципиально иной ситуации: сужение линии поглощения легких атомов щелочных металлов $\left({ }^{7} \mathrm{Li},{ }^{23} \mathrm{Na}\right)$, находящихся в атмосфере тяжелых инертных газов $(\mathrm{Xe}, \mathrm{Kr})$, при росте интенсивности излучения. Расчеты [4] проведены на примере трехуровневой $\Lambda$ системы энергетических уровней поглощающих частиц (в данной задаче $\Lambda$-систему вполне можно использовать для моделирования легких атомов щелочных металлов) в модели газа Лоренца (предельный случай тяжелых буферных частиц $M \ll M_{b}$, где $M$ и $M_{b}-$ соответственно массы резонансной и буферной частиц). Оказалось, что эффект полевого сужения линии поглощения обусловлен, во-первых, оптической накачкой на сверхтонкие компоненты основного состояния и, во-вторных, тем, что столкновительная релаксация скоростей резонансных частиц при условии $M \ll M_{b}$ разбивается на два существенно отличающихся в $\left(M / M_{b}\right.$ раз) по продолжительности этапа: релаксация по направлению скорости (быстрый этап) и релаксация по модулю скорости (медленный этап).

Проведенные в работе [4] расчеты полевого сужения линии поглощения носят сугубо оценочный характер, поскольку выполнены для идеализированного предельного случая $M / M_{b} \rightarrow 0$. Аналитические выражения для расчета формы линии поглощения, полученные в [4], дают правильные результаты именно для этого случая и описывают максимально возможное проявление полевого сужения линии поглощения. Возникает естественный вопрос о том, как будет проявляться полевое сужение при реальном соотношении масс резонансной и буферной частиц. Решение этой задачи можно получить только численными методами. Целью настоящей работы является детальное численное исследование эффекта полевого сужения линии поглощения в случае произвольного (в рамках условия $M / M_{b} \ll 1$ ) отношения масс резонансной и буферной частиц.

\section{Исходные уравнения}

В рассматриваемой задаче для расчета линии поглощения легких атомов щелочных металлов $\left({ }^{7} \mathrm{Li},{ }^{23} \mathrm{Na}\right)$ вполне можно использовать трехуровневую модель поглощающих частиц (рис. 1). Здесь уровни $n, k-$ компоненты сверхтонкой структуры основного состояния 


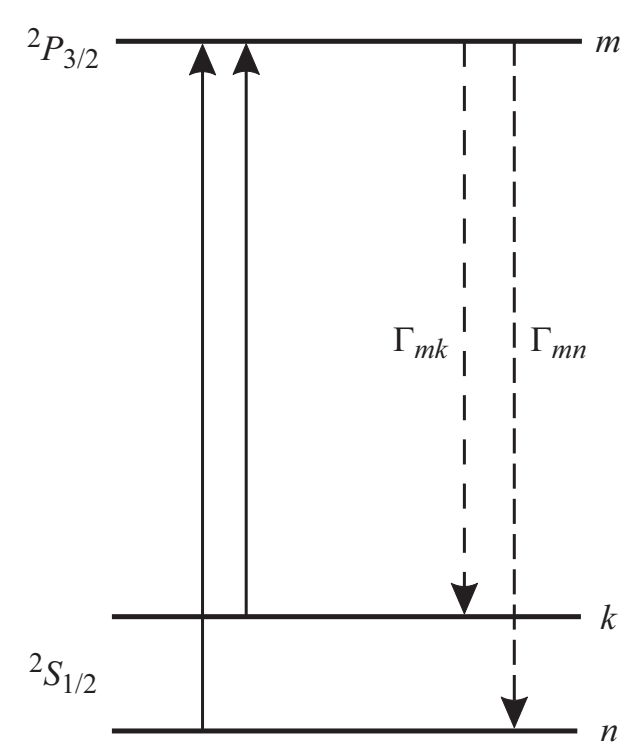

Рис. 1. Схема энергетических уровней. Прямыми стрелками обозначены переходы под действием излучения, штриховыми - спонтанные радиационные переходы.

${ }^{2} S_{1 / 2}$. Уровень $m$ соответствует возбужденному электронному состоянию ${ }^{2} P_{3 / 2}$ (для определенности будем рассматривать поглощение излучения в $D_{2}$-линиях щелочных металлов). С уровня $m$ частица радиационно релаксирует на уровни $n$ и $k$ с константами соответственно $\Gamma_{m n}$ и $\Gamma_{m k}$. Эта схема уровней хорошо отражает реальную структуру основного и первого возбужденного состояний атомов ${ }^{7} \mathrm{Li}$ и ${ }^{23} \mathrm{Na}$. Действительно, основной уровень ${ }^{2} S_{1 / 2}$ этих атомов расщеплен на две сверхтонкие компоненты. Для атомов ${ }^{7} \mathrm{Li}$ и ${ }^{23} \mathrm{Na}$ сверхтонкое расщепление основного состояния равно соответственно $\omega_{k n}=5.049 \cdot 10^{9} \mathrm{~s}^{-1}$ и $\omega_{k n}=1.113 \cdot 10^{10} \mathrm{~s}^{-1}[5]$ и сравнимо с допплеровской шириной резонансной линии. Поэтому основное состояние моделируется двумя уровнями $n$ и $k$. Для атомов ${ }^{7} \mathrm{Li}$ и ${ }^{23} \mathrm{Na}$ уровень $n$ характеризуется статистическим весом $g_{n}=3$ (полный момент атома $F=1$ ), а уровень $k-$ статистическим весом $g_{k}=5(F=2)$. Уровень $m$ (со статистическим весом $g_{m}$ ) моделирует группу уровней, представляющих собой компоненты сверхтонкой структуры возбужденного состояния ${ }^{2} P_{3 / 2}$. Такое моделирование группы уровней одним уровнем возможно потому, что для атомов ${ }^{7} \mathrm{Li}$ и ${ }^{23} \mathrm{Na}$ сверхтонкое расщепление в этом возбужденном состоянии мало по сравнению с допплеровской шириной резонансной линии. Для атомов ${ }^{7} \mathrm{Li}$ и ${ }^{23} \mathrm{Na}$ статистический вес $g_{m}=16$ при возбуждении $D_{2}$-перехода (излучением затрагивается уровень ${ }^{2} P_{3 / 2}$ с четырьмя сверхтонкими компонентами $F=0, F=1, F=2, F=3$ ).

Итак, рассмотрим газ трехуровневых поглощающих частиц (схема уровней поглощающих частиц показана на рис. 1), находящихся в смеси с буферным газом. Столкновениями между поглощающими частицами пренебрежем, полагая концентрацию буферного газа $N_{b}$ много большей концентрации поглощающего газа $N$. Взаимодействие поглощающих частиц газа с резонансным излучением в стационарных и пространственно однородных условиях описывается следующими уравнениями для распределений населенностей по скоростям $\rho_{i}(\mathbf{v})$ на уровнях $i=n, k, m$ :

$$
\begin{gathered}
S_{m}(\mathbf{v})+N\left[P_{n}(\mathbf{v})+P_{k}(\mathbf{v})\right]-\Gamma_{m} \rho_{m}(\mathbf{v})=0, \\
S_{n}(\mathbf{v})-N P_{n}(\mathbf{v})+\Gamma_{m n} \rho_{m}(\mathbf{v})=0, \\
S_{k}(\mathbf{v})-N P_{k}(\mathbf{v})+\Gamma_{m k} \rho_{m}(\mathbf{v})=0,
\end{gathered}
$$

где $N=N_{n}+N_{k}+N_{m}-$ полная концентрация поглощающих частиц, $N_{i}=\int \rho_{i}(\mathbf{v}) d \mathbf{v}-$ концентрация поглощающих частиц на уровне $i, S_{i}(\mathbf{v})-$ интегралы столкновений, $P_{n}(\mathbf{v})$ и $P_{k}(\mathbf{v})-$ вероятности поглощения излучения в единицу времени на переходах $n \rightarrow m$ и $k \rightarrow m$ в расчете на один поглощающий атом с заданной скоростью v. Для рассматриваемой трехуровневой модели частиц скорости $\Gamma_{m n}$ и $\Gamma_{m k}$ спонтанного распада уровня $m$ по каналам $m \rightarrow n$ и $m \rightarrow k$ подчиняются следующим соотношениям [6]:

$$
\Gamma_{m n}+\Gamma_{m k}=\Gamma_{m}, \quad \frac{\Gamma_{m n}}{\Gamma_{m k}}=\frac{g_{n}}{g_{k}},
$$

где $\Gamma_{m}-$ полная скорость спонтанного распада возбужденного уровня $m$. При взаимодействии поглощающих частиц с монохроматическим излучением вероятность поглощения излучения $P_{i}(\mathbf{v})$ в уравнениях (1) определяется следующим известным выражением:

$$
\begin{gathered}
N P_{i}(\mathbf{v})=\frac{B I}{\pi} Y_{i}(\mathbf{v})\left[\rho_{i}(\mathbf{v})-\frac{g_{i}}{g_{m}} \rho_{m}(\mathbf{v})\right], \\
B=\frac{\lambda^{2} \Gamma_{m}}{4 \hbar \omega} \frac{g_{m}}{g_{n}+g_{k}}, \\
Y_{i}(\mathbf{v})=\frac{\Gamma(v)}{\Gamma^{2}(v)+\left(\Omega_{i}-\mathbf{k v}\right)^{2}}, \quad \Gamma(v)=\frac{\Gamma_{m}}{2}+\gamma(v), \\
\Omega_{i}=\omega-\omega_{m i}, \quad i=n, k,
\end{gathered}
$$

где $B-$ второй коэффициент Эйнштейна для поглощения $[6] ; \omega, \lambda, \mathbf{k}$ и $I-$ частота, длина волны, волновой вектор и интенсивность монохроматического излучения; $\omega_{m i}$ - частота перехода $m \rightarrow i ; \Gamma(v)$ - однородная полуширина линии поглощения, которая в общем случае зависит от скорости и является суммой спонтанной, $\Gamma_{m} / 2$, и столкновительной, $\gamma(v)$, полуширин.

Для атомов щелочных металлов, находящихся в атмосфере инертных буферных газов, сечения столкновительных переходов $n \rightarrow k, k \rightarrow n$ между компонентами сверхтонкой структуры основного состояния очень малы - на 5-10 порядков меньше газокинетических сечений $[7$, с. 220]. Имея в виду эту ситуацию, далее будем рассматривать случай отсутствия столкновительного обмена между сверхтонкими компонентами $n$ и $k$, т.е. будем полагать, что интегралы столкновений $S_{n}(\mathbf{v})$ и $S_{k}(\mathbf{v})$ в (1) обусловлены только упругим рассеянием. Тем 
самым выполняются условия для проявления оптической накачки уровней сверхтонкой структуры основного состояния.

Далее будем рассматривать случай, когда легкие атомы щелочных металлов ${ }^{7} \mathrm{Li}$ и ${ }^{23} \mathrm{Na}$ находятся в атмосфере тяжелого инертного газа Хе. В этом случае для описания столкновений резонансных частиц с буферными частицами вполне подходит модель газа Лоренца, применимая при условии $M / M_{b} \ll 1$, где $M$ и $M_{b}$ - соответственно массы резонансной и буферной частиц. Действительно, для легких атомов щелочных металлов ${ }^{7} \mathrm{Li}$ и ${ }^{23} \mathrm{Na}$ условие $M / M_{b} \ll 1$ выполнено, если они находятся в атмосфере тяжелого инертного газа $\mathrm{Xe}\left(M / M_{b}=0.053\right.$ для атомов ${ }^{7} \mathrm{Li}$ в Хе и $M / M_{b}=0.175$ для атомов ${ }^{23} \mathrm{Na}$ в Хе). Для лоренцевского газа $\left(M / M_{b} \ll 1\right)$ при упругом рассеянии интегралы столкновений в уравнениях (1) имеют следующий вид $[8,9]$ :

$$
\begin{aligned}
S_{i}(\mathbf{v})= & \frac{M}{M_{b}} \frac{1}{v^{2}} \frac{d}{d v}\left[v^{2} v_{i}(v)\left(v+\frac{v_{T}^{2}}{2} \frac{d}{d v}\right) \rho_{i}(\mathbf{v})\right] \\
& +N_{b} v \int \sigma_{i}(v, \theta)\left[\rho_{i}\left(\mathbf{v}^{\prime}\right)-\rho_{i}(\mathbf{v})\right] d \mathbf{n}^{\prime}
\end{aligned}
$$

где

$$
\begin{gathered}
v_{T}=\sqrt{\frac{2 k_{B} T}{M}}, \quad \cos \theta=\mathbf{n n}^{\prime}, \quad \mathbf{n}=\frac{\mathbf{v}}{v}, \quad \mathbf{n}^{\prime}=\frac{\mathbf{v}^{\prime}}{v}, \\
v^{\prime}=v=|\mathbf{v}|, \quad i=n, k, m .
\end{gathered}
$$

Здесь $v_{T}$ - наиболее вероятная скорость поглощающих частиц; $k_{B}-$ постоянная Больцмана; $T-$ температура; $\mathbf{v}$ и $\mathbf{v}^{\prime}$ - скорости поглощающей частицы до и после столкновения соответственно; $\sigma_{i}(v, \theta)$ - сечение упругого $\left(v^{\prime}=v\right)$ рассеяния на угол $\theta$ поглощающей частицы в состоянии $i$ на буферной частице; $v_{i}(v)-$ транспортная частота столкновений поглощающей частицы в состоянии $i$ с буферными частицами. Для газа Лоренца транспортная частота $v_{i}(v)$ отвечает за столкновения, изменяющие только направление скорости, но не ее величину. Заметное изменение абсолютной величины скорости легких поглощающих частиц происходит лишь в результате $M_{b} / M \gg 1$ столкновений, в то время как направление скорости меняется уже в одном столкновении. Дифференциальный и интегральный члены в (4) описывают соответственно изменение абсолютного значения и направления скорости легких поглощающих частиц при их столкновениях с тяжелыми буферными частицами.

Поглощающие атомы ${ }^{7} \mathrm{Li}$ и ${ }^{23} \mathrm{Na}$ на разных подуровнях сверхтонкой структуры основного состояния имеют практически одинаковые потенциалы взаимодействия в столкновениях с атомами буферного газа. Поэтому с большой точностью можно считать, что транспортные частоты столкновений на сверхтонких компонентах $n$ и $k$ равны между собой:

$$
v_{k}(v)=v_{n}(v)
$$

Для дальнейших расчетов удобно перейти к уравнениям для распределений населенностей по модулю скорости $\rho_{i}(v)$. Подставим интеграл столкновений (4) в уравнения (1) и проинтегрируем по направлениям скорости $\mathbf{v}($ по $\mathbf{n} / 4 \pi)$. В итоге можно получить следующие уравнения:

$$
\begin{gathered}
\Gamma_{m n} \rho_{m}(v)=N P_{n}(v) \\
-\frac{M}{M_{b}} \frac{1}{v^{2}} \frac{d}{d v}\left[v^{2} v_{n}(v)\left(v+\frac{v_{T}^{2}}{2} \frac{d}{d v}\right) \rho_{n}(v)\right], \\
\Gamma_{m k} \rho_{m}(v)=N P_{k}(v) \\
-\frac{M}{M_{b}} \frac{1}{v^{2}} \frac{d}{d v}\left[v^{2} v_{k}(v)\left(v+\frac{v_{T}^{2}}{2} \frac{d}{d v}\right) \rho_{k}(v)\right], \\
\sum_{i=n, k, m} v_{i}(v)\left[v+\frac{v_{T}^{2}}{2} \frac{d}{d v}\right] \rho_{i}(v)=0,
\end{gathered}
$$

где

$$
\begin{gathered}
\rho_{i}(v) \equiv \int \rho_{i}(\mathbf{v}) \frac{d \mathbf{n}}{4 \pi}, \quad P_{j}(v) \equiv \int P_{j}(\mathbf{v}) \frac{d \mathbf{n}}{4 \pi}, \\
i=n, k, m, \quad j=n, k .
\end{gathered}
$$

Последнее уравнение в (7) приведено в [8] для бесструктурных частиц и в [9] для двухуровневых частиц. Оно отражает вытекающее из уравнений (1) соотношение $S_{m}(\mathbf{v})+S_{n}(\mathbf{v})+S_{k}(\mathbf{v})=0$.

\section{Поглощение слабоинтенсивного излучения}

Для нахождения формы линии поглощения резонансных частиц ограничимся условием слабой интенсивности излучения, для чего должно выполняться условие

$$
\varkappa \equiv \frac{B I}{\pi \Gamma\left(\Gamma_{m}+v_{m}^{t r}\right)} \ll 1 .
$$

Здесь мы ввели среднюю транспортную частоту столкновений $v_{i}^{\mathrm{tr}}$, являющуюся результатом усреднения (см. ниже) частоты столкновений $v_{i}(v)$. Величина $\varkappa$ имеет смысл параметра насыщения: он характеризует степень выравнивания населенностей у частиц с резонансными скоростями (при $\left.\Omega_{i}=\mathbf{k v}\right)$.

Отметим здесь, что при ограничении (9) на интенсивность излучения возможно выполнение условия

$$
\varkappa_{0} \equiv \frac{B I}{\pi \Gamma v_{n}^{\operatorname{tr}}} \frac{M_{b}}{M} \gg 1,
$$

означающего, что скорость вынужденных переходов $B I / \pi \Gamma$ может быть достаточно велика для того, чтобы в распределениях населенностей по модулю скорости $v$ на уровнях $n, k$ возникали изотропные неравновесные структуры (скорость их столкновительной релаксации равна $\left.v_{n}^{\text {tr }} M / M_{b}\right)$. Именно этим обстоятельством - возникновением изотропных неравновесных структур на 
сверхтонких компонентах основного состояния с ростом интенсивности излучения - обусловлен эффект полевого сужения линии поглощения.

Найдем вероятность поглощения $P_{i}(v)$ в условиях (9). При ограничении (9) на интенсивность излучения в формуле (3) для $P_{i}(\mathbf{v})$ можно пренебречь населенностью $\rho_{m}(\mathbf{v})$ и, кроме того, считать амплитуду анизотропной части распределения $\rho_{i}(\mathbf{v})$ на уровнях $i=n, k$ малой по сравнению с амплитудой изотропной части. Тогда

$$
N P_{i}(\mathbf{v})=\frac{B I}{\pi} Y_{i}(\mathbf{v}) \rho_{i}(v), \quad i=n, k,
$$

где $\rho_{i}(v)$ - изотропная часть распределения $\rho_{i}(\mathbf{v})$. Проинтегрировав (11) по направлениям скорости $\mathbf{v}$, найдем вероятность поглощения излучения в единицу времени на переходе $i \rightarrow m$ частицей с фиксированным модулем скорости $v$ :

$$
N P_{i}(v)=\frac{B I}{2 \pi k} \frac{\psi_{i}(v)}{v} \rho_{i}(v)
$$

где

$$
\psi_{i}(v)=\arctan \frac{k v+\Omega_{i}}{\Gamma(v)}+\arctan \frac{k v-\Omega_{i}}{\Gamma(v)}, \quad i=n, k .
$$

Для полной интегральной вероятности поглощения излучения

$$
P=4 \pi \int_{0}^{\infty} v^{2}\left[P_{n}(v)+P_{k}(v)\right] d v
$$

с помощью (12) находим

$$
P=\frac{2 B I}{k N} \int_{0}^{\infty} v\left[\psi_{n}(v) \rho_{n}(v)+\psi_{k}(v) \rho_{k}(v)\right] d v
$$

Нормированный на максимальное значение контур линии поглощения газа атомов определяется формулой

$$
F=\frac{P}{P_{\max }}
$$

где $P_{\text {max }}$ - максимальное значение $P$.

Найдем связь между населенностями $\rho_{n}(v)$ и $\rho_{k}(v)$ в формуле (15) для вероятности поглощения излучения. Из третьего уравнения в (7), пренебрегая населенностью $\rho_{m}(v)$ (при условии (9) имеем $\left.\rho_{m}(v) \ll \rho_{n}(v), \rho_{k}(v)\right)$ и учитывая (6), получаем следующее уравнение:

$$
\left[v+\frac{v_{T}^{2}}{2} \frac{d}{d v}\right]\left[\rho_{n}(v)+\rho_{k}(v)\right]=0 .
$$

Применяя условие нормировки $N_{n}+N_{k} \simeq N$ (здесь учтено принятое нами приближение $\left.N_{m} \ll N\right)$, отсюда находим:

$$
\begin{gathered}
\rho_{n}(v)+\rho_{k}(v)=N W(v), \\
W(v)=\left(\sqrt{\pi} v_{T}\right)^{-3} \exp \left(-\frac{v^{2}}{v_{T}^{2}}\right),
\end{gathered}
$$

где $W(v)$ - распределение Максвелла.

Формула (15) для вероятности поглощения излучения с учетом выражения (18) принимает вид

$$
P=\frac{2 B I}{k} \int_{0}^{\infty} v\left[\psi_{k}(v) W(v)+\left[\psi_{n}(v)-\psi_{k}(v)\right] \frac{\rho_{n}(v)}{N}\right] d v
$$

Из этой формулы, в частности, видно, что для двухуровневых частиц (при $\omega_{k n} \rightarrow 0$ и, как следствие, при $\left.\psi_{n}(v)=\psi_{k}(v)\right)$ ширина линии поглощения не зависит от интенсивности излучения (при ограничении (9) на интенсивность излучения).

В выражении (19) для вероятности поглощения излучения находится одна неизвестная функция - распределение населенности по модулю скорости $\rho_{n}(v)$. Найдем уравнение для определения населенности $\rho_{n}(v)$. Сумма первых двух уравнений в (7) с учетом (6) дает

$$
\begin{aligned}
& \Gamma_{m} \rho_{m}(v)=N\left[P_{n}(v)+P_{k}(v)\right] \\
& -\frac{M}{M_{b}} \frac{1}{v^{2}} \frac{d}{d v}\left[v^{2} v_{n}(v)\left(v+\frac{v_{T}^{2}}{2} \frac{d}{d v}\right)\left[\rho_{n}(v)+\rho_{k}(v)\right]\right] .
\end{aligned}
$$

При максвелловском распределении суммы населенностей по модулю скорости (18) дифференциальный член в (20) равен нулю. Поэтому уравнение (20) принимает вид

$$
\Gamma_{m} \rho_{m}(v)=N\left[P_{n}(v)+P_{k}(v)\right] .
$$

Далее из первого уравнения в (7), используя (21), (12), (18), (2), получаем следующее дифференциальное уравнение для определения населенности $\rho_{n}(v)$ :

$$
\begin{gathered}
\frac{B I}{2 \pi k v}\left\{\left[w_{n} \psi_{k}(v)+w_{k} \psi_{n}(v)\right] \rho_{n}(v)-w_{n} \psi_{k}(v) N W(v)\right\} \\
=\frac{M}{M_{b}} \frac{1}{v^{2}} \frac{d}{d v}\left[v^{2} v_{n}(v)\left(v+\frac{v_{T}^{2}}{2} \frac{d}{d v}\right) \rho_{n}(v)\right]
\end{gathered}
$$

где

$$
w_{i}=\frac{g_{i}}{g_{n}+g_{k}}, \quad i=n, k
$$

Для численного решения задачи уравнение (22) целесообразно привести к следующему виду (здесь штрих в верхнем индексе означает дифференцирование по $v$ ):

$$
\begin{aligned}
v \rho_{n}^{\prime \prime}(v) & +\left[2+\frac{2 v^{2}}{v_{T}^{2}}+\frac{v v_{n}^{\prime}(v)}{v_{n}(v)}\right] \rho_{n}^{\prime}(v) \\
& +\left\{\frac{6 v}{v_{T}^{2}}+\frac{2 v^{2}}{v_{T}^{2}} \frac{v_{n}^{\prime}(v)}{v_{n}(v)}\right. \\
& \left.-\frac{Q}{v_{T}} \frac{v_{n}^{\operatorname{tr}}}{v_{n}(v)}\left[w_{n} \psi_{k}(v)+w_{k} \psi_{n}(v)\right]\right\} \rho_{n}(v) \\
& +\frac{Q}{v_{T}} \frac{v_{n}^{\operatorname{tr}}}{v_{n}(v)} w_{n} \psi_{k}(v) N W(v)=0
\end{aligned}
$$


где введен безразмерный параметр

$$
Q=\frac{B I}{\pi k v_{T} v_{n}^{\mathrm{tr}}} \frac{M_{b}}{M} .
$$

Решение уравнения (24) должно удовлетворять следующим граничным условиям:

$$
\rho_{n}^{\prime}(0)=0,\left.\quad \rho_{n}\left(v_{0}\right)\right|_{v_{0} \gg v_{T}}=0 .
$$

\section{Численный анализ полевого сужения линии поглощения}

Дифференциальное уравнение (24) в общем виде можно решить только численно. Для выполнения дальнейших расчетов необходимо знать транспортную частоту столкновений $v_{n}(v)$ атомов ${ }^{7} \mathrm{Li}$ и ${ }^{23} \mathrm{Na}$ в основном состоянии с буферными частицами Хе. Связь транспортной частоты столкновений $v_{i}(v)$ в $(24)$ с характеристиками элементарного акта рассеяния дается [9] известной формулой:

$$
v_{i}(v)=\frac{\mu}{M} \frac{N_{b} \bar{v}_{b}}{\sqrt{\pi} v^{3}} \int_{0}^{\infty} u^{2} \exp \left(-\frac{u^{2}+v^{2}}{\bar{v}_{b}^{2}}\right) F(u v) \sigma_{i}(u) d u,
$$

где

$$
\begin{gathered}
F(u v)=\frac{2 u v}{\bar{v}_{b}^{2}} \cosh \left(\frac{2 u v}{\bar{v}_{b}^{2}}\right)-\sinh \left(\frac{2 u v}{\bar{v}_{b}^{2}}\right), \\
\mu=\frac{M M_{b}}{M+M_{b}}, \quad \bar{v}_{b}=\sqrt{\frac{2 k_{B} T}{M_{b}}}
\end{gathered}
$$

$u$ - величина относительной скорости резонансной и буферной частиц до столкновения; $\sigma_{i}(u)-$ транспортное сечение рассеяния поглощающей частицы в состоянии $i$ на буферной частице. Сечения $\sigma_{i}(u)$ рассчитывались нами по точным формулам классической механики (см., например, [10,11]) с использованием неэмпирических (рассчитанных $a b$ initio) потенциалов взаимодействия для систем сталкивающихся частиц $\mathrm{Li}-\mathrm{Xe}$ и $\mathrm{Na}-\mathrm{Xe}$ [12]. Таблично заданные потенциалы взаимодействия интерполировались кубическими сплайнами.

На рис. 2 показаны рассчитанные по формуле (27) зависимости от скорости $v$ транспортных частот столкновений $v_{n}(v)$ для атомов ${ }^{7} \mathrm{Li}{ }^{23} \mathrm{Na}$ в буферном газе Хе.

Средняя транспортная частота столкновений $v_{i}^{\text {tr }}$ определяется выражением

$$
\begin{aligned}
v_{i}^{t r} & =\frac{2}{v_{T}^{2}} \int(\mathbf{e v})^{2} W(\mathbf{v}) v_{i}(v) d \mathbf{v} \\
& =\frac{8}{3 \sqrt{\pi}} \frac{\mu}{M} \frac{N_{b}}{u_{T}^{5}} \int_{0}^{\infty} u^{5} \exp \left(-\frac{u^{2}}{u_{T}^{2}}\right) \sigma_{i}(u) d u,
\end{aligned}
$$

где $u_{T}=\left(2 k_{B} T / \mu\right)^{1 / 2}-$ наиболее вероятная скорость относительного движения поглощающей и буферной

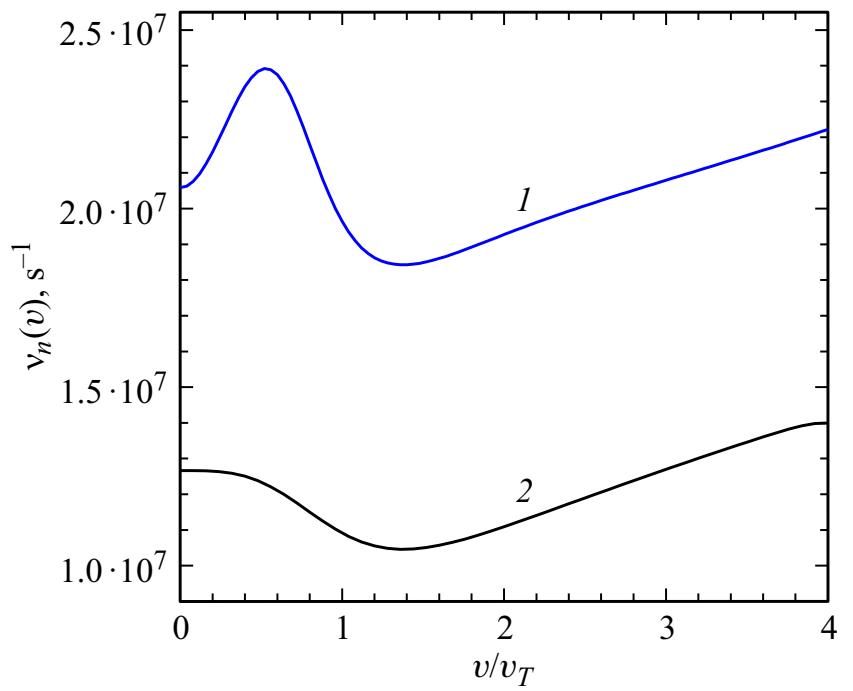

Рис. 2. Зависимость транспортной частоты столкновений $v_{n}(v)$ от модуля скорости $v$ резонансных атомов при температуре $T=300 \mathrm{~K}$ и давлении буферного газа ксенона $p_{\text {Хе }}=1$ Torr для систем сталкивающихся частиц ${ }^{7} \mathrm{Li}-\mathrm{Xe}(1)$ и ${ }^{23} \mathrm{Na}-\mathrm{Xe}(2)$. Расчет по потенциалам [12].

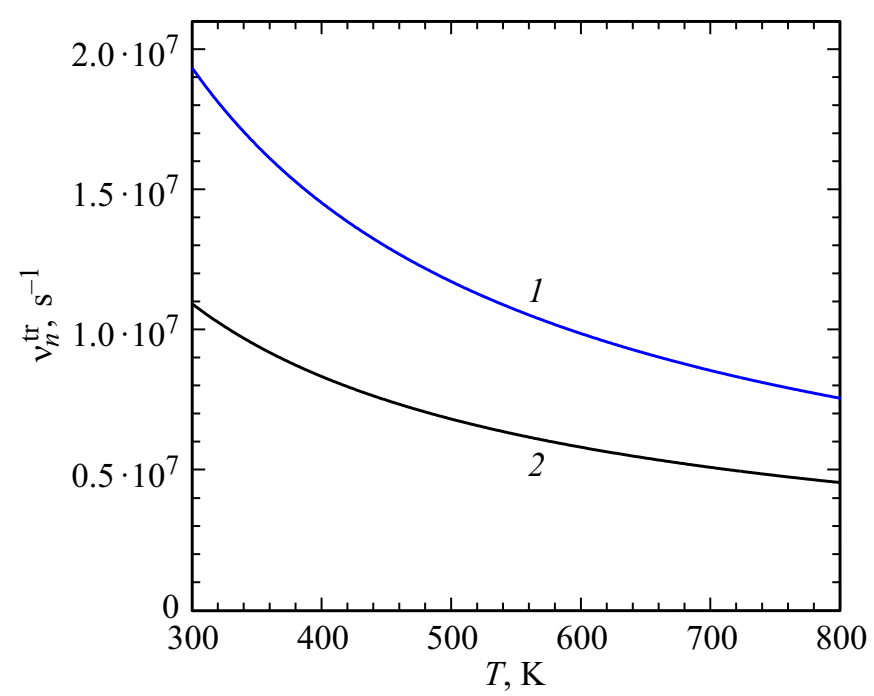

Рис. 3. Температурная зависимость средних транспортных частот столкновений атомов ${ }^{7} \mathrm{Li}(1)$ и ${ }^{23} \mathrm{Na}(2)$ в основном состоянии при их столкновении с атомами Хе при давлении газа ксенона $p_{\mathrm{Xe}}=1$ Torr. Расчет по потенциалам [12].

частиц; е - единичный вектор в произвольно выбранном направлении. Частота столкновений $v_{i}^{\text {tr }}$ связана простой формулой с коэффициентом диффузии $D_{i}$ частиц в состоянии $i[9,13]$ :

$$
D_{i}=\frac{v_{T}^{2}}{2 v_{i}^{\mathrm{tr}}} .
$$

Результаты расчетов средних транспортных частот столкновений $v_{n}^{\text {tr }}$ для атомов ${ }^{7} \mathrm{Li}$ и ${ }^{23} \mathrm{Na}$ в основном состоянии, находящихся в буферной среде атомов ксенона, показаны на рис. 3. 
Используя формулы (16), (19), (24), мы численно исследовали контур линии поглощения атомов ${ }^{7} \mathrm{Li}$ и ${ }^{23} \mathrm{Na}$ в инертном буферном газе Хе. Для атомов лития и натрия, согласно данным сайта NIST [14], скорости спонтанного распада возбужденного состояния ${ }^{2} P_{3 / 2}$ равны $\Gamma_{m}=3.69 \cdot 10^{7} \mathrm{~s}^{-1}$ и $\Gamma_{m}=6.16 \cdot 10^{7} \mathrm{~s}^{-1}$ соответственно, длины волн $D_{2}$-линии равны $\lambda=670.8 \mathrm{~nm}$ и $\lambda=589.0 \mathrm{~nm}$ соответственно. Анализ показывает, что влияние зависимости $\gamma(v)$ на эффект сужения линии поглощения незначительно и им можно пренебречь из-за того, что рассматривается случай большого допплеровского уширения. Именно поэтому при расчете контура линии поглощения мы пренебрегли зависимостью однородной полуширины линии поглощения $\Gamma(v)$ от скорости $v$ и в формуле (13) для $\psi_{i}(v)$ полагали $\Gamma(v)=\Gamma=$ const. Конкретные значения величин $\Gamma=\Gamma_{m} / 2+\gamma$ для разных систем сталкивающихся частиц $\mathrm{Li}-\mathrm{Xe}$ и $\mathrm{Na}-\mathrm{Xe}$ определялись по данным [15] для коэффициентов ударного уширения $\beta$ линии поглощения $(\beta=12.01 \mathrm{MHz} /$ Torr для атомов $\mathrm{Li} \mathrm{в}$ атмосфере $\mathrm{Xe}$ и $\beta=9.78 \mathrm{MHz} /$ Torr для атомов $\mathrm{Na}$ в атмосфеpe Xe).

Рисунки 4 и 5 иллюстрируют изменение формы и сужение линии поглощения атомов ${ }^{7} \mathrm{Li}$ и ${ }^{23} \mathrm{Na}$ в буферном газе Хе при росте интенсивности излучения. В качестве отстройки $\Omega$ частоты излучения здесь введена величина

$$
\Omega=\omega-\omega_{0}, \quad \omega_{0}=\frac{\omega_{m n}+\omega_{m k}}{2} .
$$

Частота $\omega_{0}$ соответствует среднему арифметическому значению частот переходов $\omega_{m n}$ и $\omega_{m k}$. Из рис. 4 и 5 видно, что с ростом интенсивности излучения частота максимума поглощения атомов ${ }^{7} \mathrm{Li}$ и ${ }^{23} \mathrm{Na}$ немного сдвигается в коротковолновую сторону. Для атомов ${ }^{7} \mathrm{Li}$ при температуре $T=300 \mathrm{~K}$ и давлении буферного газа ксенона $p_{\mathrm{Xe}}=0.002$ Torr ширина линии на полувысоте уменьшается в 1.20 раза при росте интенсивности излучения от $1 \mu \mathrm{W} / \mathrm{cm}^{2}$ до $2.5 \mathrm{~mW} / \mathrm{cm}^{2}$ (рис. 4). Для атомов ${ }^{23} \mathrm{Na}$ при $T=600 \mathrm{~K}$ и $p_{\text {Хе }}=0.01$ Torr ширина линии на полувысоте уменьшается в 1.29 раза при росте интенсивности излучения от $1 \mu \mathrm{W} / \mathrm{cm}^{2}$ до $6 \mathrm{~mW} / \mathrm{cm}^{2}$ (рис. 5). Сужение наиболее сильно проявляется при допплеровском уширении линии поглощения (при $\Gamma \ll k v_{T}$, низкое давление буферного газа). В случае однородного уширения (при $\Gamma \gg k v_{T}$, высокое давление буферного газа) эффект сужения линии отсутствует. Величина эффекта сужения также зависит от температуры. Для атомов ${ }^{7} \mathrm{Li}$ сужение проявляется сильнее при низких температурах $(T \approx 300 \mathrm{~K})$, а для атомов ${ }^{23} \mathrm{Na}$, наоборот, при повышенных температурах $(T \approx 600 \mathrm{~K})$.

На рис. 6 и 7 показаны рассчитанные на основе формул (16), (19), (24) ширины линии поглощения на полувысоте $\Gamma_{w}$ для атомов ${ }^{7} \mathrm{Li}$ и ${ }^{23} \mathrm{Na}$ в зависимости от интенсивности излучения при различных значениях давления буферного газа Хе. Из рисунков

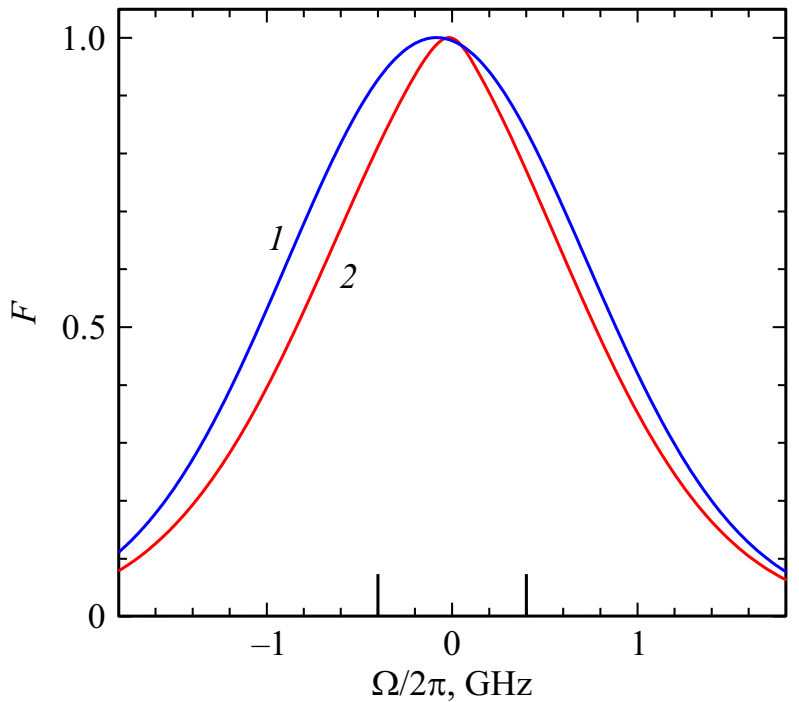

Рис. 4. Нормированный на максимальное значение контур линии поглощения атомов ${ }^{7} \mathrm{Li}$ в буферном газе Хе при $T=300 \mathrm{~K}$, $p_{\mathrm{Xe}}=0.002$ Torr; $1-I=1 \mu \mathrm{W} / \mathrm{cm}^{2}, 2-I=2.5 \mathrm{~mW} / \mathrm{cm}^{2}$. Вертикальными отрезками на оси абсцисс обозначены частоты, резонансные переходам $m-n$ и $m-k$.

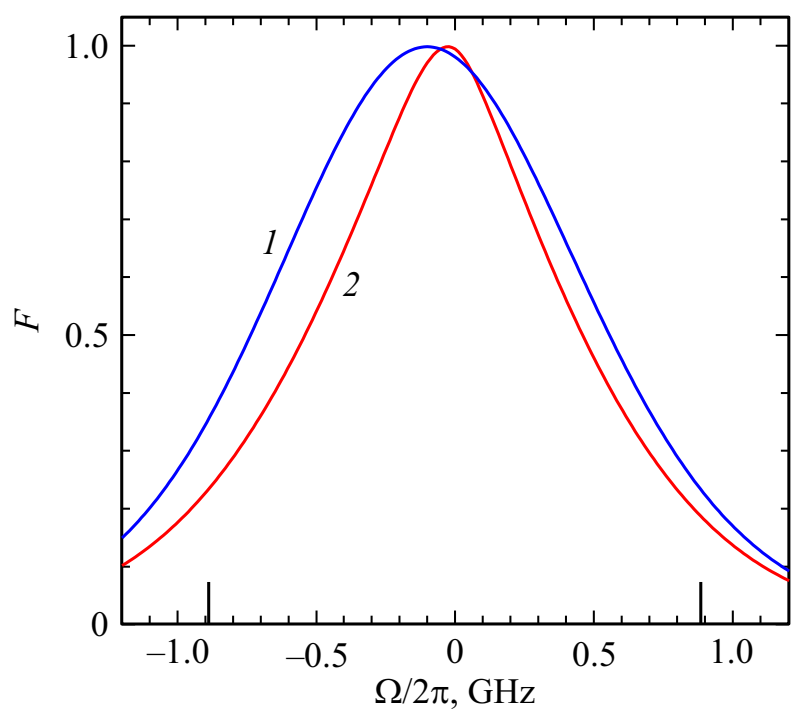

Рис. 5. Нормированный на максимальное значение контур линии поглощения атомов ${ }^{23} \mathrm{Na}$ в буферном газе Хе при $T=600 \mathrm{~K}, p_{\text {Хе }}=0.01$ Torr; $1-I=1 \mu \mathrm{W} / \mathrm{cm}^{2}, 2-$ $I=6 \mathrm{~mW} / \mathrm{cm}^{2}$. Вертикальными отрезками на оси абсцисс обозначены частоты, резонансные переходам $m-n$ и $m-k$.

видно, что ширина линии монотонно уменьшается с ростом интенсивности излучения (максимальное значение интенсивности излучения на рис. 6 и 7 ограничено условием (9) так, чтобы параметр насыщения $\varkappa$ не превышал значения 0.3). При одном и том же значении интенсивности излучения ширина линии поглощения тем меньше, чем меньше давление буферного газа. 


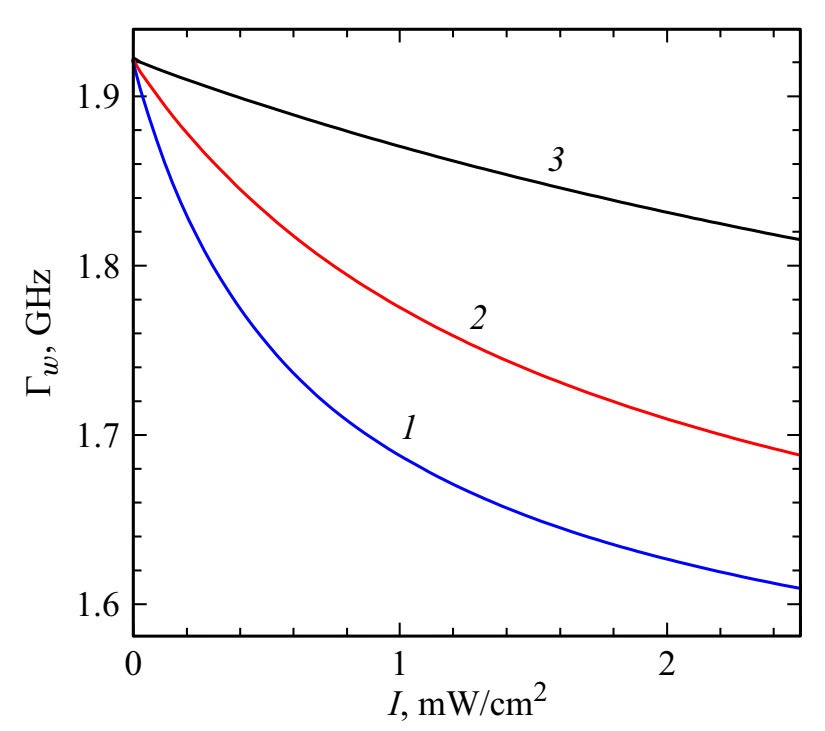

Рис. 6. Зависимость ширины линии поглощения атомов ${ }^{7} \mathrm{Li}$ от интенсивности излучения при различных значениях давления буферного газа Хе, $T=300 \mathrm{~K} ; p_{\mathrm{Xe}}=0.002(1)$, $p_{\mathrm{Xe}}=0.005(2), p_{\mathrm{Xe}}=0.02$ Torr $(3)$.

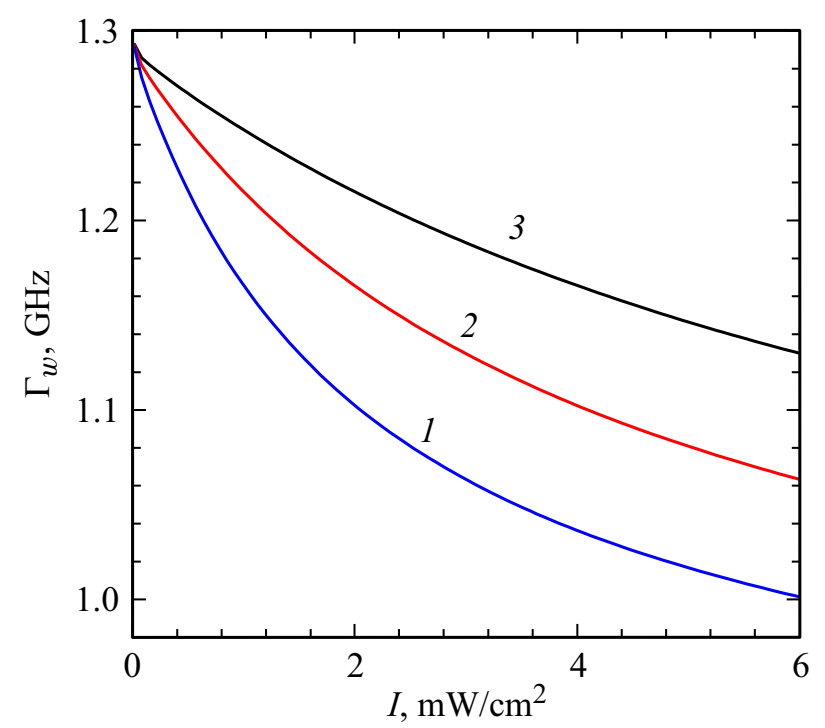

Рис. 7. Зависимость ширины линии поглощения атомов ${ }^{23} \mathrm{Na}$ от интенсивности излучения при различных значениях давления буферного газа Хе, $T=600 \mathrm{~K} ; p_{\mathrm{Xe}}=0.01$ (1), $p_{\mathrm{Xe}}=0.02(2), p_{\mathrm{Xe}}=0.04$ Torr $(3)$.

Для выяснения физической причины возникновения полевого сужения линии поглощения рассмотрим, как изменяются распределения населенностей $\rho_{n}(v)$ и $\rho_{k}(v)$ с ростом интенсивности излучения. На рис. 8 показаны распределения населенностей по модулю скорости на сверхтонких компонентах $n$ и $k$ основного состояния атомов ${ }^{7} \mathrm{Li}$ в буферном газе Хе при отстройке частоты излучения $\Omega / 2 \pi=-0.7 \mathrm{GHz}$ и остальных параметрах таких же, как на рис. 4. Линия поглощения при параметрах рис. 8 допплеровски уширена $\left(\Gamma / k v_{T}=0.0024\right)$.
В допплеровском предельном случае $\left(\Gamma \ll k v_{T}\right)$ с излучением на уровне $i=n, k$ взаимодействуют лишь те атомы, у которых абсолютная величина скорости $v \geq\left|\Omega_{i}\right| / k$, т.е. функция взаимодействия излучения с атомами $\psi_{i}(v)(13)$ имеет вид ступеньки, начинающейся при $v=\left|\Omega_{i}\right| / k$ :

$$
\psi_{i}(v)=\left\{\begin{array}{l}
0, \quad \text { если } 0 \leq v<\left|\Omega_{i}\right| / k, \\
\pi, \quad \text { если } v \geq\left|\Omega_{i}\right| / k .
\end{array}\right.
$$

Полная вероятность поглощения $P(15)$ при учете (32) пропорциональна сумме интегралов от функций $v \rho_{n}(v)$
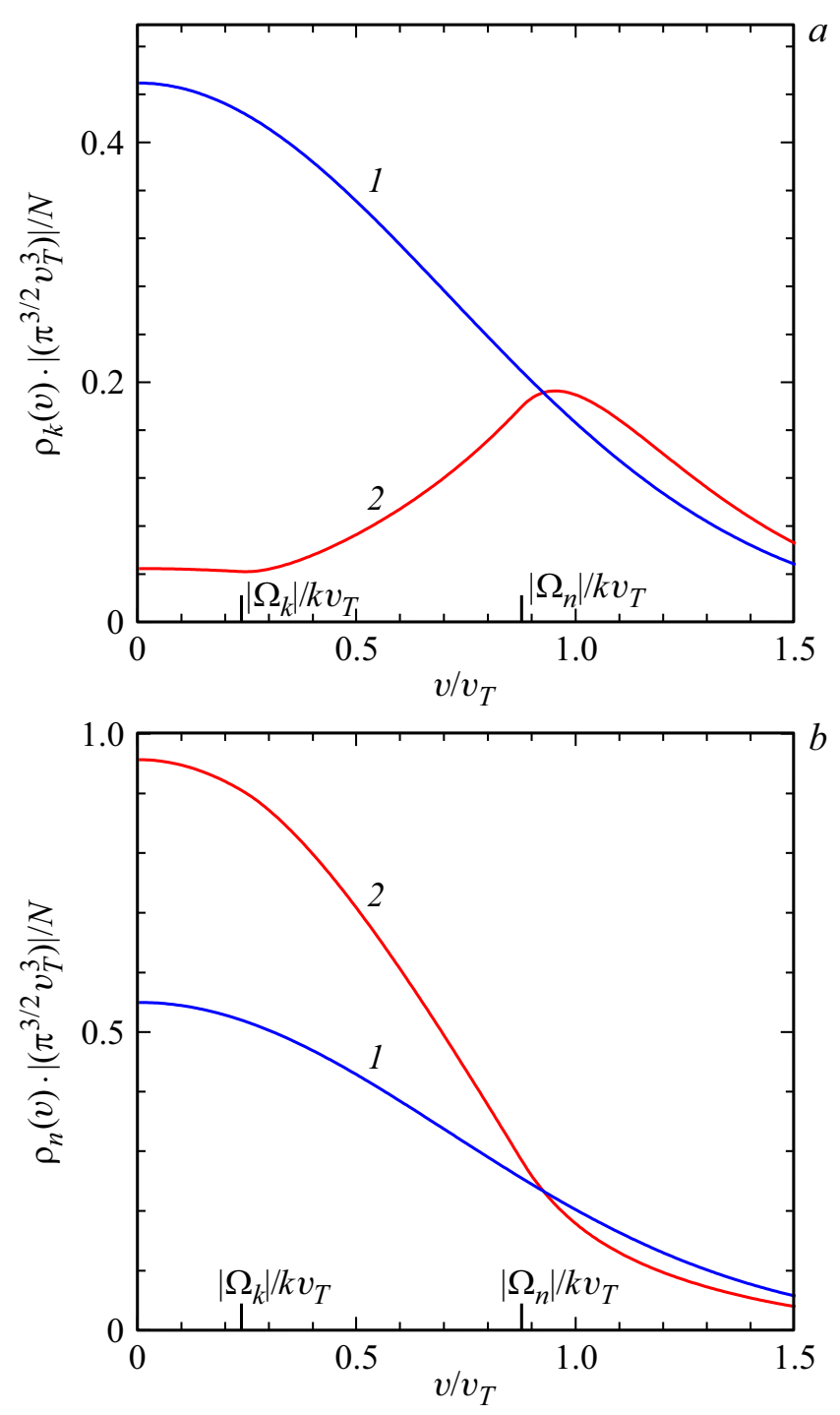

Рис. 8. Распределения населенностей по модулю скорости на сверхтонких уровнях $k(a)$ и $n(b)$ основного состояния атомов ${ }^{7} \mathrm{Li}$ в буферном газе Хе при $T=300 \mathrm{~K}, p_{\text {Хе }}=0.002$ Torr, $\Omega / 2 \pi=-0.7 \mathrm{GHz} ; 1-I=1 \mu \mathrm{W} / \mathrm{cm}^{2}, 2-I=2.5 \mathrm{~mW} / \mathrm{cm}^{2}$. Вертикальными отрезками на осях абсцисс обозначены абсолютные величины скорости $v$, равные $\left|\Omega_{n}\right| / k$ и $\left|\Omega_{k}\right| / k$. 
и $v \rho_{k}(v)$ :

$$
P \propto \int_{\left|\Omega_{n}\right| / k}^{\infty} v \rho_{n}(v) d v+\int_{\left|\Omega_{k}\right| / k}^{\infty} v \rho_{k}(v) d v
$$

Рассмотрим качественно распределение населенностей на уровнях $n$ и $k$ (рис. 8). Вследствие оптической накачки на уровень $k$ «перекачиваются» с уровня $n$ (через уровень $m$ ) частицы со скоростями $v \geq\left|\Omega_{n}\right| / k$, а на уровень $n$,перекачиваются“ с уровня $k$ частицы со скоростями $v \geq\left|\Omega_{k}\right| / k$. В интервал скоростей $\left|\Omega_{k}\right| / k \leq v \leq\left|\Omega_{n}\right| / k$ на уровне $k$ могут попасть только частицы, уже находящиеся на уровне $k$, причем приход частиц в этот интервал возможен только за счет упругих столкновений на уровне $k$ и скорость этого прихода равна $v_{n}^{\operatorname{tr}} M / M_{b}$. При низкой интенсивности излучения, такой, что $\varkappa_{0} \ll 1$, никакого отклонения от максвелловского распределения по скоростям на уровнях $n$ и $k$ не происходит (кривые 1 на рис. 8). Ситуация, однако, резко изменяется при достаточно большой интенсивности излучения, такой, что $\varkappa_{0} \gg 1$ : населенность $\rho_{k}(v)$ в интервале скоростей $\left|\Omega_{k}\right| / k \leq v \leq\left|\Omega_{n}\right| / k$ обедняется и „не успевает“ наполниться за счет столкновений, а населенность $\rho_{n}(v)$ в этом же интервале растет (кривые 2 на рис. 8).

Из сравнения распределений 1 и 2 на рис. 8 с учетом выражения (33) для $P$ следует, что при прочих равных условиях вероятность поглощения для распределений 2 будет меньше, чем для распределений 1. Это и означает, другими словами, сужение линии поглощения при переходе от распределений 1 к распределениям 2, т.е. сужение линии поглощения при росте интенсивности излучения.

\section{Заключение}

В настоящей работе численно исследован эффект сужения линии поглощения легких атомов щелочных металлов ${ }^{7} \mathrm{Li}$ и ${ }^{23} \mathrm{Na}$, находящихся в атмосфере тяжелого инертного газа Хе, при росте интенсивности излучения. При анализе использованы кинетические уравнения, упрощенные в соответствии с приближением $M / M_{b} \ll 1$. В этом приближении столкновительная релаксация скоростей резонансных частиц разбивается на два существенно различных по продолжительности этапа: релаксация по направлениям скорости (быстрый этап) и релаксация по модулю скорости (медленный этап). Для пар сталкивающихся частиц ${ }^{7} \mathrm{Li}-\mathrm{Xe} \mathrm{и}$ ${ }^{23} \mathrm{Na}-\mathrm{Xе} \mathrm{соотношение} M / M_{b} \ll 1$ удовлетворительно выполняется.

Численный анализ показывает, что для атомов ${ }^{7} \mathrm{Li}$ и ${ }^{23} \mathrm{Na}$ ширина линии на полувысоте может уменьшиться в $1.2-1.3$ раза при росте интенсивности излучения от $1 \mu \mathrm{W} / \mathrm{cm}^{2}$ до нескольких $\mathrm{mW} / \mathrm{cm}^{2}$. Полевое сужение наиболее сильно проявляется при низких давлениях буферного газа $p_{\mathrm{Xe}}=0.002-0.02$ Torr. Полевое сужение линии поглощения возникает из-за сильного изменения распределения населенностей по модулю скорости на сверхтонких компонентах $n$ и $k$ основного состояния резонансных атомов с ростом интенсивности излучения при условии $M / M_{b} \ll 1$.

\section{Финансирование работы}

Исследование выполнено за счет средств субсидии на финансовое обеспечение выполнения государственного задания (проект № АААА-А17-117052210003-4) в Институте автоматики и электрометрии Сибирского отделения РАН.

\section{Конфликт интересов}

Авторы заявляют, что у них нет конфликта интересов.

\section{Список литературы}

[1] Раутиан С.Г., Смирнов Г.И., Шалагин А.М. Нелинейные резонансы в спектрах атомов и молекул. Новосибирск: Наука, 1979. 312 с.

[2] Демтрёдер В. Лазерная спектроскопия: Основные принципы и техника эксперимента. М.: Наука, 1985. 608 с.; Demtröder $W$. Laser Spectroscopy: Basic Concepts and Instrumentation. Berlin, Heidelberg: Springer-Verlag, 2003. $1008 \mathrm{p}$.

[3] Летохов В.С., Чеботаев В.П. Нелинейная лазерная спектроскопия сверхвысокого разрешения. М.: Наука, 1990. 512 c.; Letokhov V.S., Chebotayev V.P. Nonlinear Laser Spectroscopy. Berlin: Springer-Verlag, 1977. 466 p.

[4] Гельмуханов Ф.Х., Пархоменко А.И. // ЖЭТФ. 1995. Т. 107. № 6. C. 1853;

Gel'mukhanov F.Kh., Parkhomenko A.I. // JETP. 1995. V. 80. N 6. P. 1029.

[5] Радциг А.А., Смирнов Б.М. Параметры атомов и атомных ионов: Справочник. М.: Энергоатомиздат, 1986. 344 с.; Radzig A.A., Smirnov B.M. Reference Data on Atoms, Molecules, and Ions. Berlin, Heidelberg: Springer-Verlag, 1985. $463 \mathrm{p}$.

[6] Собельман И.И. Введение в теорию атомных спектров. М.: Наука, 1977. 320 c.; Sobel'man I.I. Introduction to the Theory of Atomic Spectra. Oxford, New York: Pergamon Press, 1972. $626 \mathrm{p}$.

[7] Happer W. // Rev. Mod. Phys. 1972. V. 44. N 2. P. 169. doi 10.1103/RevModPhys.44.169

[8] Лифиии Е.М., Питаевский Л.П. Физическая кинетика. М.: Наука, 1979. 528 c.; Lifshitz E.M., Pitaevskii L.P. Physical Kinetics. Oxford, New York, Paris: Pergamon Press, 1981. $452 \mathrm{p}$.

[9] Gel'mukhanov F.Kh., Ilichov L.V., Shalagin A.M. // Physica A. 1986. V. 137. N 3. P. 502. doi 10.1016/0378-4371(86)90092-0

[10] Ферцигер Джс., Капер Г. Математическая теория процессов переноса в газах. М.: Мир, 1976. 556 c.; Ferziger J.H., Kaper H.G. Mathematical Theory of Transport Processes in Gases. Amsterdam, London: North-Holland Publishing Company, 1972.

[11] Mason E.A., McDaniel E.W. Transport properties of ions in gases. N.Y., Toronto: John Wiley \& Sons, 1988. 560 p. 
[12] Galbis E., Douady J., Jacquet E., Giglio E., Gervais B. // J. Chem. Phys. 2013. V. 138. N 1. P. 014314. doi 10.1063/1.4773019

[13] Rautian S.G., Shalagin A.M. Kinetic Problems of Nonlinear Spectroscopy. Amsterdam, N.Y: Elsevier Science Publ. Comp., 1991. 439 p.

[14] Электронный ресурс. Режим доступа: https://www.nist.gov/pml/atomic-spectra-database

[15] Allard N., Kielkopf J. // Rev. Mod. Phys. 1982. V. 54. N 4. P. 1103. doi 10.1103/RevModPhys.54.1103 